\title{
A NEWAPPROACH FOR NUCLEAR REACTOR ANALYSIS BASED ON COMPLEX NETWORK THEORY
}

\author{
E. Cervi ${ }^{\mathrm{a}}$, A. Cammi ${ }^{1 \mathrm{a}}$, E. Zio \\ aPolitecnico di Milano, Department of Energy, Nuclear Engineering Division, Via La Masa 34, 20156, \\ Milan, Italy
}

\begin{abstract}
In this work, complex network theory is applied for the first time in the field of nuclear reactor physics to present a new approach for the evaluation of the multiplication factor of a nuclear system. The approach describes the random walk of a neutron in a network representative of the nuclear system. The network is consituted by multiple layers, each one representing a type of reaction (scattering, fission and capture) and each layer is constituted by different nodes, each one representing a different spatial position in the nuclear system. The probability of a neutron to jump from a node to another is governed by the material cross sections. A correction procedure is also proposed to account for non-reaction probability, obtaining a good agreement between the predictions of the proposed method and of Monte Carlo simulation.

The outcome of this work constitutes a starting point for further research on the application of complex network theory to the field of nuclear reactor physics, while, at the same time, the experience retrieved from the application of complex networks may give useful insight for the improvement of classical approaches to nuclear reactor analysis.
\end{abstract}

KEYWORDS: Complex Networks, Reactor Physics, Neutronics.

\section{Introduction}

Complex networks represent an interdisciplinary research area which, over the years, has gathered the interest from physicists, engineers, social scientists, computer scientists, biologists and many others. Complex network structures describe a large plethora of systems of scientific and technical interest. Chemical and biological systems, financial, social and communication networks, and spreading of epidemics are just few examples (Barabási, 2016).

Complex network theory represents a simple and yet effective approach to describe and understand the structure of many complex systems of different nature. As anticipated, it has been applied in many research fields, such as chemistry (Hayat, 2017; Orlova et al., 2018), biology (Duran-Nebreda and Bassel, 2017; Fionda, 2018), epidemiology (Yuan et al., 2013; Sun et al., 2014; Schimit and Pereira, 2018), social sciences (Raducha and Gubiec, 2017; Zareie and Sheikhahmadi, 2018), computer science (Yang et al., 2013; Gan et al., 2014) and criminology (Calderoni et al. 2017).

On the other hand, reactor physics is traditionally studied by means of point kinetics (Hamieh et Saidinezhad, 2012; Cervi et al., 2018; Cervi and Cammi, 2018; Holschuh and Marcum, 2018), neutron diffusion theory (Aufiero et al., 2014; Cervi et al., 2017), neutron transport theory (Fiorina et al., 2017; Tramm et al., 2018) and Monte Carlo method (Leppänen et al., 2013; Chiesa et al., 2016). However, to the best of the authors knowledge, up to now, there is no application to the field of nuclear reactor physics.

In nuclear systems, reactions take place between the neutrons and the nuclei of the fuel, coolant and structural materials. Notably, in scattering reactions, neutrons bounce on nuclei, changing their momentum and kinetic energy; in captures, they are absorbed from a fertile or coolant nucleus, disappearing from the neutronics balance of the system; in fission reactions, a neutron is absorbed from a fissile nucleus, which splits into two smaller fragments, emitting other neutrons (Lamarsh, 1969). Neutrons and nuclei can be seen as the costituents of a network, interacting with each other through reactions of scattering, capture, and fission.

In this view, complex network theory may constitute a useful approach to nuclear reactor physics, sheding light on new aspects or providing innovative and efficient ways to study nuclear systems. Thanks to its limited

\footnotetext{
1 antonio.cammi@polimi.it (A.Cammi)
} 
computational requirements, the proposed approach could be useful for core design optimization, especially in pre-conceptual design phases in which several changes are made and a fast-running tool is needed for their assessment. In addition, complex network theory has been succesfully applied to stability analysis (Liu and Tan, 2013), control theory (Zañudo et al., 2017) and optimization of the number and position of measurement instruments and detectors (Leitold et al., 2017). These applications are also of interest in the nuclear field, thus justifying the development of a first approach to nuclear reactor analysis using complex network theory. In this framework, the purpose of this paper is to lay down a path between complex network theory and reactor physics, providing a preliminary evaluation of the potentialities of this method in nuclear engineering applications and defining a baseline approach for the analysis of nuclear reactors, which is fundamental as a starting point for more detailed analyses.

A fuel pin of different reactor types (pressurized water reactor, PWR, sodium fast reactor, SFR, and lead fast reactor, LFR) is modelled from a complex network theory perspective and a simple procedure is set up for the evaluation of the main neutronics parameters of a nuclear reactor. More in details, the fuel pin is discretized as a lattice of nodes, and the system multiplication factor and Doppler reactivity coefficient are evaluated by considering the random walk of a neutron in this complex network.

The present work is organized as follows. In Section 2, the reader is introduced to the basic concepts of complex network theory. In Section 3, the modelling approach and the considered case study are introduced, while in Section 4 the results are presented and compared to Monte Carlo simulation to assess their validity. In addition, in Section 5, a correction procedure is proposed, accounting for the non-reaction probability of the different materials, in order to improve the accuracy of the method in non-uniform multiplying media. In the same section, the corrected approach is tested against Monte Carlo simulation and compared to the uncorrected one. Conclusions and future perspectives of research are given in Section 6.

\section{Fundamentals of complex network theory}

In this section, the basic concepts of complex network theory are provided, to introduce the nuclear commuity to the topic and guide the reader in understanding its formalism. For a deeper insight into complex network theory, the reader is referred to specialized textbooks (e.g., Newman, 2010; van Steen, 2010; Barabási, 2016).

\subsection{Nodes and links}

To understand a complex system, the first thing is to understand how its parts are related to each other. A graph of the network is well suited for this, to describe the interactions between the network components. To this aim, a new branch of mathematics, the so called complex network theory, was developed. According to complex network theory, a network can be described with an array of nodes, representative of the network components, and of links (or edges), representative of the relations between the components.

An example is provided by Fig. 1, showing a protein-protein interaction network. Each protein constitutes a node of the network, while all the possible bindings between them constitute the links. Even if the nature of the nodes and of their interactions may change (e.g., in computer or social networks), the same graph representation can be used.

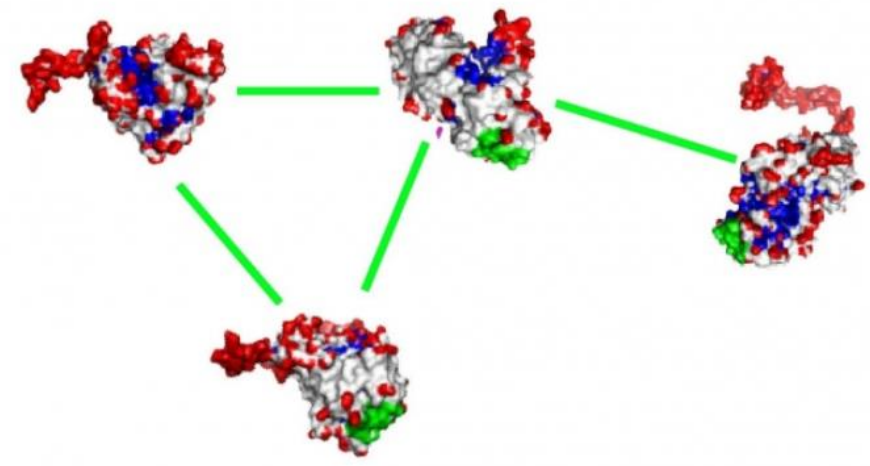

Figure 1. Protein-protein interaction network (Barabási, 2016). 
Most networks of scientific interest are weighted, i.e., their links can have in general different weights. In a protein network, weights may represent the probability of a binding between two molecules. The topology of a weighted network can be described by a so called adjacency matrix $\boldsymbol{A}$, whose generic element $a_{i j}$ represents the weight of the link going from the node $i$ to the node $j$. In case of an unweighted network, all the elements of $\boldsymbol{A}$ are equal to one, and links represent merely qualitative interactions, without providing any quantitative information.

Finally, complex networks can be distinguished between directed and undirected networks, depending on whether their links have a specified direction or not (see Fig. 2). The protein network shown in Fig. 1 is undirected, while phone calls, for example, where one person calls the other, can be thought as a directed network.
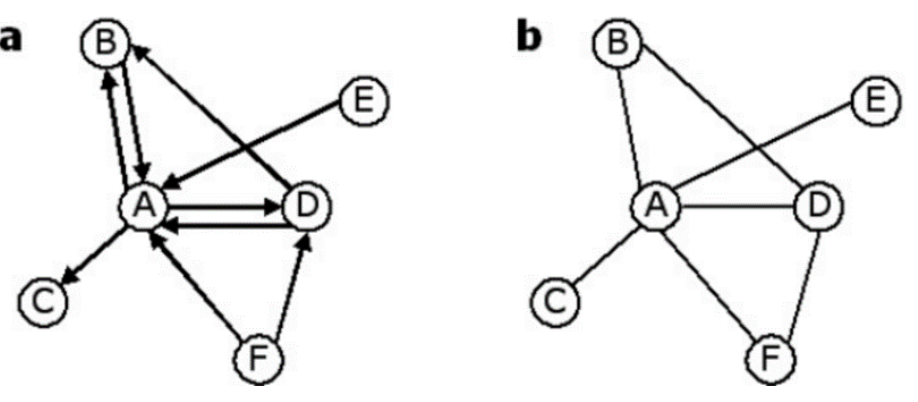

Figure 2. Directed (a) vs. undirected (b) networks.

\subsection{Node centrality}

In complex systems, some elements may be more important than others (e.g., a specific protein may undergo more reactions then other molecules). In complex network theory, the importance of a node is also called centrality. Several centrality indicators can be found in literature for the analysis of a network, depending on the specific nature of a given system (Newman, 2010; van Steen, 2010).

A possible way to evaluate node centrality is to study the path of a random walker through the network. Due to the stochastic nature of the neutron transport process, this technique can be particularly useful for nuclear applications. For this reason, this method is discussed in the present section.

In a weighted network, a random walker can move from a node $i$ to another node $j$ with probability defined as follows:

$$
p_{i j}=\frac{a_{i j}}{\sum_{j} a_{i j}}
$$

i.e., as the ratio between the weight of the link from $i$ to $j$ and the sum of the weights of all the links going from $i$ to the other nodes. The probabilities $p_{i j}$ form an $N$ x $N$ transition matrix $\boldsymbol{P}$, where $N$ is the number of nodes of the network.

A state probability vector is also defined:

$$
\pi_{t}=\left(\pi_{1}, \pi_{2}, \ldots, \pi_{N}\right)_{t}
$$

whose elements $\pi_{i}$ represent the probability that the random walker is in the node $i$ at time $t$. Indicating with $[t, t+1]$ a generic time interval between two events (i.e., two random steps), the state probability vector evolves according to the following equation:

$$
\pi_{t+1}=\boldsymbol{P} \pi_{t}
$$

A network is said to be strictly connected if, for every pair of nodes $i$ and $j$, there always exists a sequence of links going from $i$ to $j$. If this condition is verified, it can be proved that there is a unique stationary state probability vector $\pi$, which is strictly positive (i.e., $\pi_{i}>0$ for every $i$ ) and satisfies the following equation (Piccardi, 2011): 
The time index is omitted in Eq. (4), since in stationary conditions the state probability vector is no longer dependent on time. It is stressed that $\boldsymbol{\pi}$ is a probability vector, meaning the random walker can still move from a node to another, but the probability to be in a generic node $i$ is constant in time. The stationary elements $\pi_{i}$ represent the fraction of time spent by the random walker in the node $i$ in stationary conditions and, as a consequence, can be assumed as a centrality indicator of that node.

\section{Case study}

\subsection{System geometry}

A simplified two-dimensional fuel cell, constituted by a fuel pin and surrounding coolant, is considered as a case study for the present work. The pin radius is $0.475 \mathrm{~cm}$, while the half pitch is $0.63 \mathrm{~cm}$. This geometry is discretized in a certain number of spatial nodes, as shown in Fig. 3.

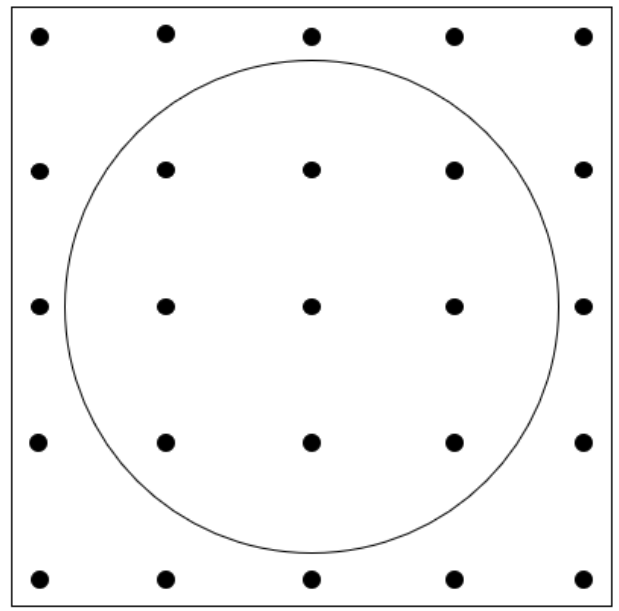

Figure 3. Geometry of the fuel cell and discretization in nodes. For simplicity, only 25 nodes are shown in this picture, but a higher number of nodes are used troughout this work.

Each node represents a position which could be occupied by a neutron during its path and is characterized by a set of cross sections depending on the specific material at that node. Homogenized, group-constant cross sections for the fuel and the coolant can be obtained by Monte Carlo simulation: thus, the nodes falling in the fuel region are characterized by the fuel cross sections and the nodes falling in the coolant region are characterized by the coolant cross sections. In this work, spatial effects within material regions are neglected, i.e., nodes belonging to the same region have the same set of cross sections.

\subsection{Neutron random walk}

Given the cross sections, the probability of each reaction can be calculated. In particular, for each node:

$$
\begin{gathered}
\text { fission probability }=p_{f}=\frac{\sum_{\text {fission }}}{\sum_{\text {total }}} \\
\text { scattering probability }=p_{s}=\frac{\sum_{\text {scattering }}}{\sum_{\text {total }}} \\
\text { capture probability }=p_{c}=\frac{\sum_{\text {capture }}}{\sum_{\text {total }}}
\end{gathered}
$$

These probabilities govern the neutron random walk from a node to another. In this paper, the total, scattering, capture and fission cross sections of the fuel and of the coolant are calculated using the Monte Carlo reactor 
physics code Serpent 2 (Leppänen et al., 2015), adopting the JEFF-3.1.1 libraries for the cross section data (Santamarina et al., 2009).

Since three different types of reaction may occur (fission, scattering and capture) a three-layer network is constructed, with each layer representing a reaction type (see Fig. 4). For each spatial position, there is a node in the fission layer, a node in the scattering layer and a node in the capture layer, representing respectively a fission, a scattering and a capture occurring at that given position.

Consider the random walk of a neutron, which is initially in the scattering layer:

- The neutron may induce a fission in a fissile nucleus, thus making a transition from the scattering layer to the fission layer with probability $p_{f}$. Upon fission, the original neutron ceases to exist, and new neutrons are generated at that position. Then, the random walk proceeds considering the path of these neutrons, returning to the scattering layer with a probability equal to 1 .

- The neutron may be captured by a fertile nucleus, transferring from the scattering layer to the capture layer with probability $p_{c}$. Also in this case, the neutron ceases to exist once it is captured. The path of a new neutron is, then, considered in the same position where the capture occurred. This choice is made to have the least impact on the neutron random walk, restarting it where it is interrupted. Other options have been considered (e.g., starting new neutrons at fission nodes), verifying that the adopted choice is the best in terms of result accuracy.

- The neutron can be scattered with probability $p_{s}$. In this case, the neutron moves from a node to another in the scattering layer. Considering four discrete directions in two dimensions, as represented in Fig. 4 , and assuming isotropic scattering, the neutron can move to one of the four adjacent nodes with probability $p_{s} / 4$.

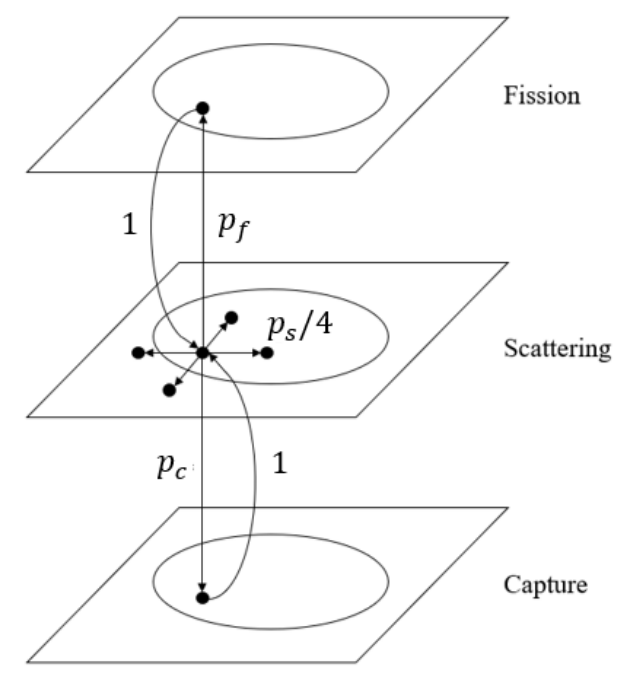

Figure 4. Three-layer network adopted to describe the fuel pin.

Summarizing, a nuclear reaction is sampled with a probability which depends on the corresponding cross section of the material at the node position and, if scattering occurs, a direction is sampled from a uniform distribution (for the isotropic scattering assumption). At this point the length of the neutron free path should be sampled to determine where the following collision occurs. The case study considered in the present work is constituted by two different materials, each with its own macroscopic total cross section. Therefore, the free path length distribution is different in each node and when a neutron moves from a node to another, the path length sampled in the first node may not be statistically valid in the second, since cross sections could change. A common way to address this issue is to resample the free path length each time the neutron reaches a new material. In practice, this requires the comparison between the sampled length and the distance to the nearest surface of interface between two materials, which is the idea at the basis of the ray-tracing method adopted in Monte Carlo simulations (Haghighat, 2014).

In a complex network theory approach, this would require the definition of a scattering matrix containing all the possible links between all the nodes of the network. Each value of the matrix should depend not only on 
the distance between two points, but also on all the different materials that the neutron encounters in the path from the initial to the successive point. However, such operation would be impractical, especially for complex three-dimensional geometries and heterogeneous media constituted by different materials. To overcome this issue, an approximation is made, assuming that the free path is always equal to one step and that neutrons undergo another reaction in the adjacent node. In other words, this is equivalent to neglecting the non-reaction probability, imposing that a reaction always occurs whenever a neutron moves from a node to another. For the sake of conciseness, this will be referred to as the "one-step approximation" through the remainder of the manuscript. In the following sections, the limits of this approximation are discussed and a correction procedure is proposed, to account for non-reaction probability and improve the accuracy of the approach.

At this point, all the elements of the network topology have been introduced. Summarizing, for each spatial position there are three nodes (one for each layer), representing the three possible reactions (scattering, capture and fission). Hence, each node represents a given type of reaction, occurring at a given point of the system. On the other hand, the links of the network represent the reaction probabilities, which depend on the cross sections according to Eqs. (5) to (7). This network is weighted, since the weight of the links changes depending on the material and reaction type, and directed, since neutrons have a specified flight direction.

In addition, the defined network is strictly connected, since given two nodes, there is always a path between them, in both directions (i.e., from the first node to the second as well as from the second to the first). Note that the fission nodes of the coolant region cannot be reached at all by neutrons, since fissions do not occur there. These nodes are simply not seen by the random walker, as if they are not part of the network. However, the remaining portion of the network, were the random walk actually takes place, is strictly connected. Due to strict connection, the random walk of a neutron can be described using Eq. (4), here reported for the sake of readability:

$$
\pi=P \pi
$$

Recalling Section 2.2, the elements of the vector $\pi$ (i.e., the node centralities) represent the stationary probabilities to observe a specific reaction type at a given spatial position of the system.

Note that the random walk statistics are entirely captured by the transition matrix $\boldsymbol{P}$, whose elements describe the probability of a neutron step from a node to another. In addition, according to Eq. (4), the stationary state probability vector $\pi$ can be evaluated as the eigenvector corresponding to the unit eigenvalue. Therefore, the calculation is purely deterministic and can be performed without actually simulating the neutron random walk (as opposed to the Monte Carlo approach, in which many random walks must be sampled in order to describe the neutron global behavior). For this reason, results are not affected by statistics (i.e., by the number of neutron histories) but only by the cross section errors and by the modelling assumptions.

\subsection{The global network}

The basic idea underlying the proposed approach is to use the centrality indicators $\pi_{i}$ to obtain an information on the system eigenvalue. However, while node centrality provides a local information on reaction probability, the multiplication factor depends on the global neutron balance of the system. To overcome this, starting from the network introduced in the previous section, an aggregate network is defined, constituted by only three nodes. These nodes have the role of "clusters", grouping together the fission layer nodes, the scattering layer nodes and the capture layer nodes of the original network, respectively (see Fig. 5).

As a random walk in the original network can be described by the transition matrix $\boldsymbol{P}$, in the same way a global transition matrix $\boldsymbol{U}$ can be defined for the aggregate network, whose elements $u_{i j}$ (with $i$ and $j=$ fission (f), scattering (s) or capture (c)) represent the global probability to move from a layer to another (as indicated in Fig. 5). $\boldsymbol{U}$ is a $3 \times 3$ matrix, since the original network is composed of three layers, and the following procedure can be used to evaluate it. First, a "clustering" matrix $\boldsymbol{H}$ is introduced, whose elements $h_{i k}$ are defined as:

$$
h_{i k}= \begin{cases}1 & \text { if the node } i \text { belongs to the layer } k \\ 0 & \text { otherwise }\end{cases}
$$



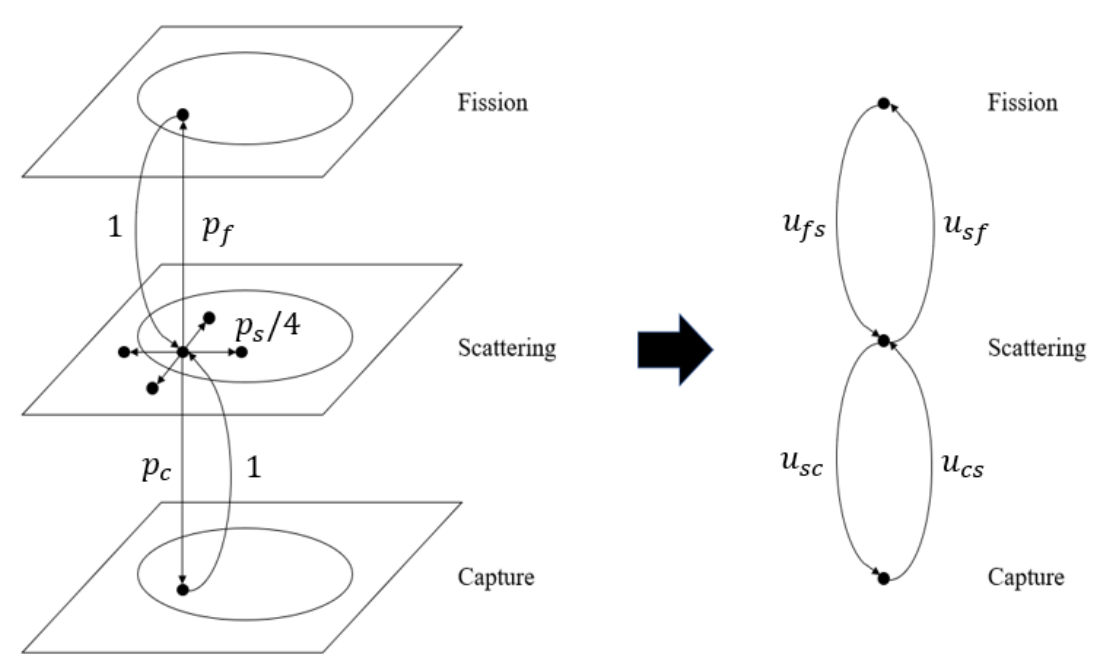

Figure 5. Global network obtained from the original one.

Following its definition, $\boldsymbol{H}$ is a $N \times 3$ matrix, which simply indicates, for each node of the original network, the corresponding layer. Its definition is necessary for the evaluation of $\boldsymbol{U}$, according to the following expression (Piccardi, 2011):

$$
\boldsymbol{U}=[\operatorname{diag}(\boldsymbol{\pi} \boldsymbol{H})]^{-1} \boldsymbol{H}^{T} \operatorname{diag}(\boldsymbol{\pi}) \boldsymbol{P} \boldsymbol{H}
$$

The matrix $\boldsymbol{U}$ is the equivalent of the transition matrix $\boldsymbol{P}$, referred to the global network instead of the original one. Thanks to Eq. (9), the spatial dependence of the reaction probability (contained in $\boldsymbol{\pi}$ and $\boldsymbol{P}$ ) is condensed in $\boldsymbol{U}$. Hence, the matrix elements $u_{i j}$ represent the global probability that a neutron undergoes a given type of reaction and therefore provide an information of the neutron behavior over the whole system. In the following section, this information is used to evaluate the system multiplication factor.

\subsection{Evaluation of the multiplication factor}

In the light of what is described in the previous sections, a neutron random walk can be described using the original network, in which the nodes of each layer represent different positions in space and using the aggregate network, representative of the global probabilities that a neutron undergoes a certain reaction, making a transition from a layer to another. Describing the neutron behavior over the entire system, the aggregate network is particularly suitable to extract information on the system eigenvalue. The expression for the evaluation of the multiplication factor is derived as follows.

During the random walk, if the system is critical, the number of neutrons produced by fissions must be equal to the number of neutrons lost by captures. Hence, the following relation must be valid:

$$
(\bar{v}-1) u_{s f}=u_{s c}
$$

where $u_{s f}$ is the global probability that the neutron induces a fission, in which one neutron is absorbed and $\bar{v}$ new neutrons are produced, while $u_{s c}$ is the global probability that the neutron is lost by capture.

Eq. (10) is satisfied only in the case of a critical system. Then, in analogy with the $k$-eigenvalue method adopted in the Monte Carlo approach, the multiplication factor $k$ is defined as the eigenvalue that makes the system stationary, bringing it back to criticality:

$$
\left(\frac{\bar{v}}{k}-1\right) u_{s f}=u_{s c}
$$

from which the multiplication factor can be obtained as: 


$$
k=\frac{\bar{v} u_{s f}}{u_{s c}+u_{s f}}
$$

\section{Results}

Once $k$ is known, the reactivity $\rho$ can be calculated as:

$$
\rho=\frac{k-1}{k}
$$

A comparison is, then, made between the reactivity calculated by the complex network theory approach and the Monte Carlo results, in order to assess the accuracy of the proposed method. A comparison is also made between the Doppler reactivity coefficients, defined as:

$$
\alpha_{D}=\frac{\rho\left(T_{2}\right)-\rho\left(T_{1}\right)}{T_{2}-T_{1}}
$$

Monte Carlo calculations are carried out using the continuous-energy code Serpent 2 (Leppänen et al., 2015) and the JEFF-3.1.1 cross section library (Santamarina et al., 2009). All the simulations are performed with 100 million neutron histories (10000 generations, 10000 neutrons per generation and one generation per batch), obtaining a $5 \mathrm{pcm}$ uncertainty on the multiplication factor. The same simulations are used to generate the homogeneized one-group cross sections for the complex network theory approach (see Eqs. (5) to (7)). A set of cross sections is generated for each material region and for each value of the fuel temperature considered for the evaluation of $\alpha_{D}$. It is also reminded that spatial effects in each material region are neglected. Therefore, nodes belonging to the same region are characterized by the same cross sections.

The following different systems are considered as case studies:

- A Pressurized Water Reactor (PWR) $\mathrm{UO}_{2}$ pin with $3.25 \%{ }^{235} \mathrm{U}$ atom enrichment;

- A Sodium Fast Reactor (SFR) MOX pin with $15 \%{ }^{239} \mathrm{Pu}$ atom enrichment;

- A Lead Fast Reactor (LFR) MOX pin with $15 \%{ }^{239} \mathrm{Pu}$ atom enrichment;

- A uniform ${ }^{235} \mathrm{U}$ medium.

In all cases, an infinite (or periodic) boundary condition is assumed. If a neutron is scattered out of the geometry boundary, it returns in the domain from the opposite side of the boundary (see Fig. 6). This is equivalent to assuming that the considered geometry is situated in an infinite medium, constituted by a periodic lattice. A total number of 400 nodes $(20 \times 20)$ is used to discretize the pin. A sensitivity study on the number of nodes has been carried out, verifying that the results are not modified by further refinements.

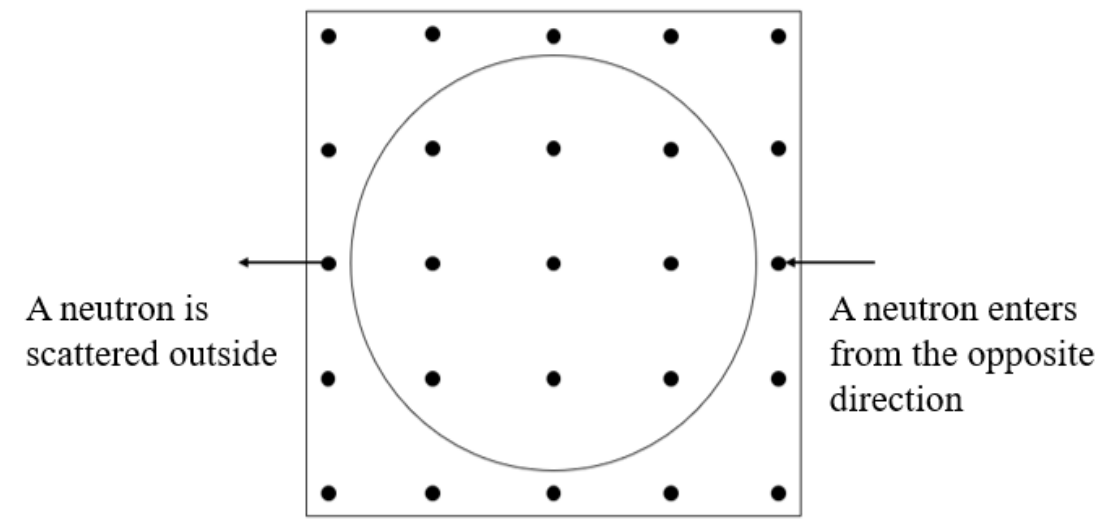

Figure 6. Periodic boundary condition (for simplicity, 25 nodes are shown in this picture, but 400 nodes are adopted for the calculations). 


\subsection{PWR fuel pin}

The multiplication factor and the Doppler reactivity coefficient of a PWR fuel pin are calculated at different fuel temperatures and their values are compared to those obtained by Monte Carlo simulation. The results are reported in Tables I to III.

Table I. Multiplication factor and reactivity difference with respect to the value obtained by Monte Carlo simulation for a PWR fuel pin, for $T_{\text {fuel }}=600^{\circ} \mathrm{C}$ and $T_{\text {coolant }}=300^{\circ} \mathrm{C}$.

\begin{tabular}{|l|l|l|}
\hline & Complex network & Monte Carlo \\
\hline$k$ & 1.32760 & $1.31253 \pm 0.00005$ \\
\hline$\rho_{C N}-\rho_{M C}$ & $865 \mathrm{pcm}$ & - \\
\hline
\end{tabular}

Table II. Multiplication factor, reactivity difference with respect to the value obtained by Monte Carlo simulation and Doppler coefficient for a PWR fuel pin, for $T_{\text {fuel }}=900^{\circ} \mathrm{C}$ and $T_{\text {coolant }}=300^{\circ} \mathrm{C}$.

\begin{tabular}{|l|l|l|}
\hline & Complex network & Monte Carlo \\
\hline$k$ & 1.31489 & $1.30022 \pm 0.00005$ \\
\hline$\rho_{C N}-\rho_{M C}$ & $858 \mathrm{pcm}$ & - \\
\hline$\alpha_{D}\left(T_{\text {fuel }}=600 \div 900{ }^{\circ} \mathrm{C}\right)$ & $-2.428 \mathrm{pcm} /{ }^{\circ} \mathrm{C}$ & $-2.404 \pm 0.020 \mathrm{pcm} /{ }^{\circ} \mathrm{C}$ \\
\hline
\end{tabular}

Table III. Multiplication factor, reactivity difference with respect to the value obtained by Monte Carlo simulation and Doppler coefficient for a PWR fuel pin, for $T_{\text {fuel }}=1200^{\circ} \mathrm{C}$ and $T_{\text {coolant }}=300^{\circ} \mathrm{C}$.

\begin{tabular}{|l|l|l|}
\hline & Complex network & Monte Carlo \\
\hline$k$ & 1.30441 & $1.28993 \pm 0.00005$ \\
\hline$\rho_{C N}-\rho_{M C}$ & $861 \mathrm{pcm}$ & - \\
\hline$\alpha_{D}\left(T_{\text {fuel }}=900 \div 1200{ }^{\circ} \mathrm{C}\right)$ & $-2.037 \mathrm{pcm} /{ }^{\circ} \mathrm{C}$ & $-2.045 \pm 0.020 \mathrm{pcm} /{ }^{\circ} \mathrm{C}$ \\
\hline
\end{tabular}

\subsection{SFR fuel pin}

For the SFR fuel pin, the results are summarized in Tables IV to VI.

Table IV. Multiplication factor and reactivity difference with respect to the value obtained by Monte Carlo simulation for a SFR fuel pin, for $T_{\text {fuel }}=600^{\circ} \mathrm{C}$ and $T_{\text {coolant }}=425^{\circ} \mathrm{C}$.

\begin{tabular}{|l|l|l|}
\hline & Complex network & Monte Carlo \\
\hline$k$ & 1.45900 & $1.47637 \pm 0.00005$ \\
\hline$\rho_{C N}-\rho_{M C}$ & $-807 \mathrm{pcm}$ & - \\
\hline
\end{tabular}

Table V. Multiplication factor, reactivity difference with respect to the value obtained by Monte Carlo simulation and Doppler coefficient for a SFR fuel pin, for $T_{\text {fuel }}=900^{\circ} \mathrm{C}$ and $T_{\text {coolant }}=425^{\circ} \mathrm{C}$.

\begin{tabular}{|l|l|l|}
\hline & Complex network & Monte Carlo \\
\hline$k$ & 1.45341 & $1.47065 \pm 0.00005$ \\
\hline$\rho_{C N}-\rho_{M C}$ & $-807 \mathrm{pcm}$ & - \\
\hline$\alpha_{D}\left(T_{\text {fuel }}=600 \div 900{ }^{\circ} \mathrm{C}\right)$ & $-0.879 \mathrm{pcm} /{ }^{\circ} \mathrm{C}$ & $-0.878 \pm 0.015 \mathrm{pcm} /{ }^{\circ} \mathrm{C}$ \\
\hline
\end{tabular}

Table VI. Multiplication factor, reactivity difference with respect to the value obtained by Monte Carlo simulation and Doppler coefficient for a SFR fuel pin, for $T_{\text {fuel }}=1200^{\circ} \mathrm{C}$ and $T_{\text {coolant }}=425^{\circ} \mathrm{C}$.

\begin{tabular}{|l|l|l|}
\hline & Complex network & Monte Carlo \\
\hline$k$ & 1.44990 & $1.46704 \pm 0.00005$ \\
\hline$\rho_{C N}-\rho_{M C}$ & $-806 \mathrm{pcm}$ & - \\
\hline$\alpha_{D}\left(T_{\text {fuel }}=900 \div 1200{ }^{\circ} \mathrm{C}\right)$ & $-0.555 \mathrm{pcm} /{ }^{\circ} \mathrm{C}$ & $-0.558 \pm 0.015 \mathrm{pcm} /{ }^{\circ} \mathrm{C}$ \\
\hline
\end{tabular}




\subsection{LFR fuel pin}

For the LFR fuel pin, the results are summarized in Tables VII to IX.

Table VII. Multiplication factor and reactivity difference with respect to the value obtained by Monte Carlo simulation for a LFR fuel pin, for $T_{\text {fuel }}=600^{\circ} \mathrm{C}$ and $T_{\text {coolant }}=440^{\circ} \mathrm{C}$.

\begin{tabular}{|l|l|l|}
\hline & Complex network & Monte Carlo \\
\hline$k$ & 1.43541 & $1.44919 \pm 0.00005$ \\
\hline$\rho_{C N}-\rho_{M C}$ & $-676 \mathrm{pcm}$ & - \\
\hline
\end{tabular}

Table VIII. Multiplication factor, reactivity difference with respect to the value obtained by Monte Carlo simulation and Doppler coefficient for a LFR fuel pin, for $T_{\text {fuel }}=900^{\circ} \mathrm{C}$ and $T_{\text {coolant }}=440^{\circ} \mathrm{C}$.

\begin{tabular}{|l|l|l|}
\hline & Complex network & Monte Carlo \\
\hline$k$ & 1.43178 & $1.44581 \pm 0.00005$ \\
\hline$\rho_{C N}-\rho_{M C}$ & $-678 \mathrm{pcm}$ & - \\
\hline$\alpha_{D}\left(T_{\text {fuel }}=600 \div 900{ }^{\circ} \mathrm{C}\right)$ & $-0.545 \mathrm{pcm} /{ }^{\circ} \mathrm{C}$ & $-0.538 \pm 0.016 \mathrm{pcm} /{ }^{\circ} \mathrm{C}$ \\
\hline
\end{tabular}

Table IX. Multiplication factor, reactivity difference with respect to the value obtained by Monte Carlo simulation and Doppler coefficient for a LFR fuel pin, for $T_{\text {fuel }}=1200^{\circ} \mathrm{C}$ and $T_{\text {coolant }}=440^{\circ} \mathrm{C}$.

\begin{tabular}{|l|l|l|}
\hline & Complex network & Monte Carlo \\
\hline$k$ & 1.42974 & $1.44376 \pm 0.00005$ \\
\hline$\rho_{C N}-\rho_{M C}$ & $-679 \mathrm{pcm}$ & - \\
\hline$\alpha_{D}\left(T_{\text {fuel }}=900 \div 1200{ }^{\circ} \mathrm{C}\right)$ & $-0.332 \mathrm{pcm} /{ }^{\circ} \mathrm{C}$ & $-0.327 \pm 0.016 \mathrm{pcm} /{ }^{\circ} \mathrm{C}$ \\
\hline
\end{tabular}

\subsection{Uniform medium}

In this section, the multiplication factor of a uniform ${ }^{235} \mathrm{U}$ medium is calculated. The value obtained with the complex network theory approach is $k_{C N}=2.26534$, while the value from Monte Carlo is $k_{M C}=$ 2.275353. The reactivity difference between the two methods is $-198 \mathrm{pcm}$.

For this simple case, the multiplication factor can be calculated also analytically. Using the one-group formulation of the neutron diffusion equation (Lamarsh, 1969), it can be easily found that:

$$
k=\frac{\bar{v} \sum_{f}}{\sum_{a}}
$$

where the absorption cross section $\sum_{a}$ is the sum of the fission and capture cross sections. It can be observed that Eq. (15) is formally equivalent to Eq. (12). In fact, substituting into Eq. (15) the same homogenized cross sections used for the complex network theory method, the predicted multiplication factor is $k_{D I F F}=2.26534$, which is exactly coincident with $k_{C N}$, independently from the number of nodes of the network.

\subsection{Discussion of results}

The results presented in the previous sections point out that the multiplication factor predicted with the complex network theory approach agrees with the Monte Carlo results within 600-900 pcm, with the exception of the uniform medium, for which the reactivity difference is lower than $200 \mathrm{pcm}$.

Good agreement is obtained between the Doppler coefficients predicted by the two approaches. This can be expected, since the homogeneized cross sections adopted in the complex network theory approach are generated from the same Monte Carlo simulations that are used for the comparison (and therefore, both the methods predict the same reactivity variations). However, considering the simplicity of the proposed approach, such agreement is still an interesting result. 
The reactivity error between the two approaches is due to different reasons, namely, the use of group-constant cross sections and the adoption of only four discretized flight directions for the scattering neutrons. However, the one-step approximation introduced in Section 3.2 is also expected to have an important role.

As explained, assuming that the neutron free path always has the same length, equal to the distance between two adjacent nodes, is equivalent to neglecting the non-reaction probability. In fact, a reaction event is imposed everytime a neutron moves from a node to another. This approximation is rigorously justified in an infinite and homogeneous multiplying medium, in which cross sections are uniform in space and, as a consequence, the random walk statistics are independent from the spatial position. Therefore, in this case, sampling the free path is unnecessary and an arbitrary free flight can be assumed without affecting results. However, in nonuniform systems, the non-reaction probability and, as a consequence, the expected free path length, change from point to point. Hence, if the medium is heterogeneous (which is the case for the major part of the systems of scientific and technical interest) the one-step approximation is a critical limit for the proposed method, constituting an important source of error. This consideration is supported by the fact that in the fuel pin cases the error is about four time higher, compared to the uniform medium test case. To overcome this issue, a correction procedure, accounting for the spatial dependene of the non-reaction probability, is proposed in the following section, in order to improve the accuracy of the proposed method without renouncing the simplicity of the one-step approximation.

\section{The $\sum_{\text {majorant }}$ correction}

As discussed in Section 4.5, the non-rection probabilities in different material regions should be accounted to improve the accuracy of the proposed approach. This issue is addressed by means of the following procedure, which is inspired to the Woodcock's delta-tracking method (Woodcock et al., 1965):

- a majorant cross section is defined as the maximum of the material total cross sections:

$$
\sum_{\text {majorant }}=\max \left\{\sum_{\text {total }}^{m}, \quad \text { where } m=\text { materials }\right\}
$$

- for each material, the reaction probabilities are redefined as follows:

$$
\begin{gathered}
p_{f}^{\prime}=\frac{\sum_{\text {total }}}{\sum_{\text {majorant }}} \frac{\sum_{\text {fission }}}{\sum_{\text {total }}}=\frac{\sum_{\text {fission }}}{\sum_{\text {majorant }}} \\
p_{s}^{\prime}=\frac{\sum_{\text {total }}}{\sum_{\text {majorant }}} \frac{\sum_{\text {scattering }}}{\sum_{\text {total }}}=\frac{\sum_{\text {scattering }}}{\sum_{\text {majorant }}} \\
p_{c}^{\prime}=\frac{\sum_{\text {total }}}{\sum_{\text {majorant }}} \frac{\sum_{\text {capture }}}{\sum_{\text {total }}}=\frac{\sum_{\text {capture }}}{\sum_{\text {majorant }}}
\end{gathered}
$$

- a non-reaction probability is defined as:

$$
p_{n r}^{\prime}=1-p_{f}^{\prime}-p_{s}^{\prime}-p_{c}^{\prime}
$$

- finally, the reaction cross sections are again redefined according to the following relations:

$$
\begin{gathered}
p_{f}^{*}=p_{f}^{\prime} \\
p_{s}^{*}=p_{s}^{\prime}+p_{n r}^{\prime} \\
p_{c}^{*}=p_{c}^{\prime}
\end{gathered}
$$

The general idea of this approach is to homogenize the medium with the introduction of the majorant cross section, so that all the materials have the same "virtual" total cross section. Then, by replacing $\sum_{t o t a l}$ with 
$\sum_{\text {majorant }}$ in Eqs. (16), (17) and (18), the reaction probabilities are corrected by the "weight" of each node, i.e. by the probability that a reaction occurs in that node. Hence, the non-reaction probability must be defined as the complement to unity of the reaction probabilities, since the sum of probabilities must be equal to 1 . Finally, the non reaction-probability is included in the scattering probability, so that the scattering layer of the network becomes not only representative of the scattered neutrons, but also of the non-reacting ones (see Fig. 7).

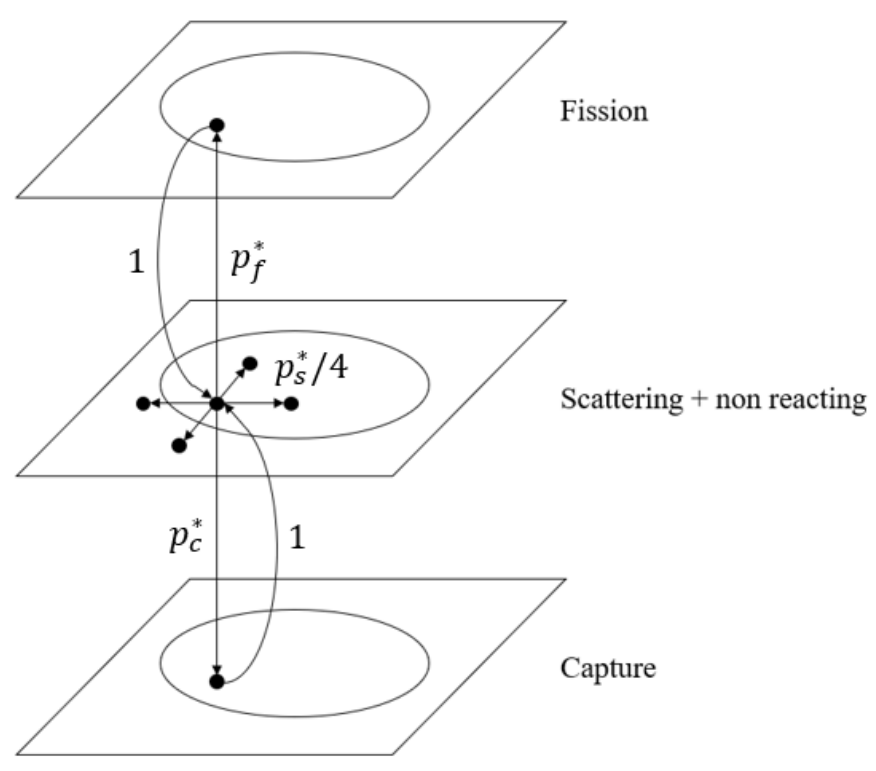

Figure 7. Complex network including non-reacting event

\subsection{PWR fuel pin}

For the LFR fuel pin, the results are summarized in Tables X to XII.

Table X. Multiplication factor and reactivity difference with respect to the value obtained by Monte Carlo simulation for a PWR fuel pin, for $T_{\text {fuel }}=600^{\circ} \mathrm{C}$ and $T_{\text {coolant }}=300^{\circ} \mathrm{C}$.

\begin{tabular}{|l|l|l|}
\hline & Complex network & Monte Carlo \\
\hline$k$ & 1.31341 & $1.31253 \pm 0.00005$ \\
\hline$\rho_{C N}-\rho_{M C}$ & $51 \mathrm{pcm}$ & - \\
\hline
\end{tabular}

Table XI. Multiplication factor, reactivity difference with respect to the value obtained by Monte Carlo simulation and Doppler coefficient for a PWR fuel pin, for $T_{\text {fuel }}=900^{\circ} \mathrm{C}$ and $T_{\text {coolant }}=300^{\circ} \mathrm{C}$.

\begin{tabular}{|l|l|l|}
\hline & Complex network & Monte Carlo \\
\hline$k$ & 1.30108 & $1.30022 \pm 0.00005$ \\
\hline$\rho_{C N}-\rho_{M C}$ & $51 \mathrm{pcm}$ & - \\
\hline$\alpha_{D}\left(T_{\text {fuel }}=600 \div 900{ }^{\circ} \mathrm{C}\right)$ & $-2.405 \mathrm{pcm} /{ }^{\circ} \mathrm{C}$ & $-2.404 \pm 0.020 \mathrm{pcm} /{ }^{\circ} \mathrm{C}$ \\
\hline
\end{tabular}

Table XII. Multiplication factor, reactivity difference with respect to the value obtained by Monte Carlo simulation and Doppler coefficient for a PWR fuel pin, for $T_{\text {fuel }}=1200^{\circ} \mathrm{C}$ and $T_{\text {coolant }}=300^{\circ} \mathrm{C}$.

\begin{tabular}{|l|l|l|}
\hline & Complex network & Monte Carlo \\
\hline$k$ & 1.29090 & $1.28993 \pm 0.00005$ \\
\hline$\rho_{C N}-\rho_{M C}$ & $58 \mathrm{pcm}$ & - \\
\hline$\alpha_{D}\left(T_{\text {fuel }}=900 \div 1200{ }^{\circ} \mathrm{C}\right)$ & $-2.020 \mathrm{pcm} /{ }^{\circ} \mathrm{C}$ & $-2.045 \pm 0.020 \mathrm{pcm} /{ }^{\circ} \mathrm{C}$ \\
\hline
\end{tabular}




\subsection{SFR fuel pin}

For the SFR fuel pin, the results are summarized in Tables XIII to XV.

Table XIII. Multiplication factor and reactivity difference with respect to the value obtained by Monte Carlo simulation for a SFR fuel pin, for $T_{\text {fuel }}=600^{\circ} \mathrm{C}$ and $T_{\text {coolant }}=425^{\circ} \mathrm{C}$.

\begin{tabular}{|l|l|l|}
\hline & Complex network & Monte Carlo \\
\hline$k$ & 1.47202 & $1.47637 \pm 0.00005$ \\
\hline$\rho_{C N}-\rho_{M C}$ & $-200 \mathrm{pcm}$ & - \\
\hline
\end{tabular}

Table XIV. Multiplication factor, reactivity difference with respect to the value obtained by Monte Carlo simulation and Doppler coefficient for a SFR fuel pin, for $T_{\text {fuel }}=900^{\circ} \mathrm{C}$ and $T_{\text {coolant }}=425^{\circ} \mathrm{C}$.

\begin{tabular}{|l|l|l|}
\hline & Complex network & Monte Carlo \\
\hline$k$ & 1.46631 & $1.47065 \pm 0.00005$ \\
\hline$\rho_{C N}-\rho_{M C}$ & $-201 \mathrm{pcm}$ & - \\
\hline$\alpha_{D}\left(T_{\text {fuel }}=600 \div 900{ }^{\circ} \mathrm{C}\right)$ & $-0.882 \mathrm{pcm} /{ }^{\circ} \mathrm{C}$ & $-0.878 \pm 0.015 \mathrm{pcm} /{ }^{\circ} \mathrm{C}$ \\
\hline
\end{tabular}

Table XV. Multiplication factor, reactivity difference with respect to the value obtained by Monte Carlo simulation and Doppler coefficient for a SFR fuel pin, for $T_{\text {fuel }}=1200^{\circ} \mathrm{C}$ and $T_{\text {coolant }}=425^{\circ} \mathrm{C}$.

\begin{tabular}{|l|l|l|}
\hline & Complex network & Monte Carlo \\
\hline$k$ & 1.46271 & $1.46704 \pm 0.00005$ \\
\hline$\rho_{C N}-\rho_{M C}$ & $-202 \mathrm{pcm}$ & - \\
\hline$\alpha_{D}\left(T_{\text {fuel }}=900 \div 1200{ }^{\circ} \mathrm{C}\right)$ & $-0.559 \mathrm{pcm} /{ }^{\circ} \mathrm{C}$ & $-0.558 \pm 0.015 \mathrm{pcm} /{ }^{\circ} \mathrm{C}$ \\
\hline
\end{tabular}

\subsection{LFR fuel pin}

For the SFR fuel pin, the results are summarized in Tables XVI to XVIII.

Table XVI. Multiplication factor and reactivity difference with respect to the value obtained by Monte Carlo simulation for a LFR fuel pin, for $T_{\text {fuel }}=600^{\circ} \mathrm{C}$ and $T_{\text {coolant }}=440^{\circ} \mathrm{C}$.

\begin{tabular}{|l|l|l|}
\hline & Complex network & Monte Carlo \\
\hline$k$ & 1.44528 & $1.44919 \pm 0.00005$ \\
\hline$\rho_{C N}-\rho_{M C}$ & $-187 \mathrm{pcm}$ & - \\
\hline
\end{tabular}

Table XVII. Multiplication factor, reactivity difference with respect to the value obtained by Monte Carlo simulation and Doppler coefficient for a LFR fuel pin, for $T_{\text {fuel }}=900^{\circ} \mathrm{C}$ and $T_{\text {coolant }}=440^{\circ} \mathrm{C}$.

\begin{tabular}{|l|l|l|}
\hline & Complex network & Monte Carlo \\
\hline$k$ & 1.44190 & $1.44581 \pm 0.00005$ \\
\hline$\rho_{C N}-\rho_{M C}$ & $-187 \mathrm{pcm}$ & - \\
\hline$\alpha_{D}\left(T_{\text {fuel }}=600 \div 900{ }^{\circ} \mathrm{C}\right)$ & $-0.541 \mathrm{pcm} /{ }^{\circ} \mathrm{C}$ & $-0.538 \pm 0.016 \mathrm{pcm} /{ }^{\circ} \mathrm{C}$ \\
\hline
\end{tabular}

Table XVIII. Multiplication factor, reactivity difference with respect to the value obtained by Monte Carlo simulation and Doppler coefficient for a LFR fuel pin, for $T_{\text {fuel }}=1200^{\circ} \mathrm{C}$ and $T_{\text {coolant }}=440^{\circ} \mathrm{C}$.

\begin{tabular}{|l|l|l|}
\hline & Complex network & Monte Carlo \\
\hline$k$ & 1.43986 & $1.44376 \pm 0.00005$ \\
\hline$\rho_{C N}-\rho_{M C}$ & $-188 \mathrm{pcm}$ & - \\
\hline$\alpha_{D}\left(T_{\text {fuel }}=900 \div 1200{ }^{\circ} \mathrm{C}\right)$ & $-0.328 \mathrm{pcm} /{ }^{\circ} \mathrm{C}$ & $-0.327 \pm 0.016 \mathrm{pcm} /{ }^{\circ} \mathrm{C}$ \\
\hline
\end{tabular}




\title{
5.4. Discussion of results
}

\begin{abstract}
The results show that the $\sum$ majorant correction leads to significant improvements in the prediction of the multiplication factor. The reactivity difference with respect to Monte Carlo simulation is reduced from about $600-900 \mathrm{pcm}$ to $50-200 \mathrm{pcm}$, similar to the uniform case. These results support the consideration made in Section 4.5, that the one-step approximation represents a major source of error for the uncorrected approach. In fact, upon application of the $\sum_{\text {majorant }}$ correction, the method's accuracy becomes comparable to the uniform case study, which is not affected by the one-step approximation.

The residual error can be imputed to the one group-approximation as well as to the discretization of the flight direction and, considering the simplicity of the method, it is surprisingly small.

A comparison between several deterministic reactor physics codes on a similar 2D pin test case can be found in Rowlands et al. (1999). An agreement of $\sim 20-360 \mathrm{pcm}$ is found with Monte Carlo results, which is compatible with the accuracy of the complex network theory approach. In addition, a comparison with the neutron transport code DRAGON5 (Hebért, 2016), in terms of accuracy and runtime, is presented in Appendix A, using the fuel pin case studies adopted in the previous sections.

Concerning computational time, the complex network theory approach requires about 20-25 seconds per simulation, using an Intel ${ }^{\circledR}$ Core $^{\circledR}$ i7-6700HQ CPU with 2 x 4 cores and a clock speed of $2.60 \mathrm{GHz}$ in serial mode. The application of the $\sum_{\text {majorant }}$ correction does not visibly affect run times, compared to the uncorrected approach.
\end{abstract}

\section{Conclusions}

In this work, an innovative approach to nuclear reactor analysis is proposed, bases on complex network theory. For demonstration purposes, a nuclear reactor fuel pin is modelled as a complex network and the multiplication factor and the Doppler reactivity coefficient are evaluated for a PWR, a SFR and a LFR fuel pin. For all the considered case studies, the predicted reactivity agrees within $600-900$ pcm with Monte Carlo simulation results. A possible source of error is the "one-node approximation", i.e., the assumption that a reaction must take place every time a neutron moves from a node to an adjacent one, neglecting non-reaction. As discussed, this approximation can be rigorously applied in infinite, homogeneous systems, in which cross sections (and, as a consequence, the mean free path) are uniform in space. On the other hand, it can significantly affect results in heterogeneous systems, in which the mean free path depends on the different materials encountered by the neutron during the free flight. To address this issue, the medium is "homogeneized" by correcting the reaction probability by a majorant cross section, allowing to account for non-reaction probability. Good agreement is obtained between the corrected approach and Monte Carlo simulation, reducing the error to about 50-200 pcm. In conclusion, this work paves, for the first time, a way for treating nuclear reactor physics by complex network theory, providing a simple and yet effective approach to estimate the neutronics parameters of a nuclear system. Complex network theory may be of interest in the nuclear field due to its possible application to control theory, stability analysis and optimization of measurement instrumentation (Liu and Tan, 2013; Gomez Tejeda Zañudo et al., 2017; Leitold et al., 2017). Therefore, the present work may constitute the first step for the development of new and accurate methods for the analysis of nuclear systems from a complex network theory perspective. In particular, the definition and the testing of a baseline appraoch is fundamental as a starting point for more detailed analyses based on this method. In addition, the limited computational requirements can be particularly interesting for core design optimization, especially in preliminary phases where specifications are subject to frequent changes and, as a consequence, a fast running tool is required. Future work could regard the extension of the present method to multiple neutron energy groups and by considering thermal-hydraulics, providing a fully coupled description of a nuclear system.

\section{A. Comparison with the DRAGON5 reactor physics code}

In this Appendix, the proposed method is compared to the deterministic neutron transport code DRAGON5 (Hebért, 2016; documentation available at http://www.polymtl.ca/phys/en/dragon-download), using the PWR, SFR and LFR fuel pin case studies. The same homogeneized one-group cross sections adopted in the complex 
network theory approach are also used in the DRAGON5 simulations, in order to carry out the comparison in the same conditions. The multiplication factor and the reactivity difference with respect to Monte Carlo obtained with DRAGON5 are presented in Table A.I. For comparison, the complex network theory errors $\rho_{C N}-\rho_{M C}$, obtained with the $\sum_{\text {majorant }}$ correction (Sections 5.1, 5.2 and 5.3), are also reported.

It can be observed that the two approaches are comparable in terms of accuracy. For each case study, the complex network theory errors ( $\sim 50-200 \mathrm{pcm})$ are slightly smaller than the DRAGON5 errors $(\sim 130-220 \mathrm{pcm})$. As far as computational times are concerned, each DRAGON5 simulation is carried out in approximately $2-3$ seconds, while the complex network theory approach requires 20-25 seconds per calculation. However, it has to be considered that the DRAGON5 code is written in FORTRAN, which is a compiled programming language, while the proposed approach is implemented in MATLAB ${ }^{\circledR}$, which is based on an interpreted language and therefore it is intrinsically slower. In principle, the complex network theory approach could be implemented using other programming languages, possibly reducing computational times.

Table A.I. Comparison between DRAGON5 and the complex network theory approach.

\begin{tabular}{|c|c|c|c|c|c|}
\hline Case & $T_{\text {fuel }}(\mathbf{K})$ & $k_{D R A G O N}$ & $\boldsymbol{k}_{M C}$ & $\begin{array}{c}\rho_{\text {DRAGON }}-\rho_{M C} \\
(\mathrm{pcm})\end{array}$ & $\begin{array}{c}\rho_{C N}-\rho_{M C} \\
(\mathbf{p c m})\end{array}$ \\
\hline \multirow[t]{3}{*}{ PWR } & 600 & 1.31023 & $1.31253 \pm 0.00005$ & -134 & 51 \\
\hline & 900 & 1.29795 & $1.30022 \pm 0.00005$ & -135 & 51 \\
\hline & 1200 & 1.28778 & $1.28993 \pm 0.00005$ & -129 & 58 \\
\hline \multirow[t]{3}{*}{ SFR } & 600 & 1.47180 & $1.47637 \pm 0.00005$ & -210 & -200 \\
\hline & 900 & 1.46600 & $1.47065 \pm 0.00005$ & -216 & -201 \\
\hline & 1200 & 1.46243 & $1.46704 \pm 0.00005$ & -215 & -202 \\
\hline \multirow[t]{3}{*}{ LFR } & 600 & 1.44441 & $1.44919 \pm 0.00005$ & -228 & -187 \\
\hline & 900 & 1.44117 & $1.44581 \pm 0.00005$ & -223 & -187 \\
\hline & 1200 & 1.43912 & $1.44376 \pm 0.00005$ & -223 & -188 \\
\hline
\end{tabular}

\section{Nomenclature}

\section{Latin symbols}

$\begin{array}{ll}a & \text { Link weight, - } \\ \boldsymbol{A} & \text { Adjacency matrix, - } \\ \boldsymbol{H} & \text { Matrix defined in Eq. (8), - } \\ k & \text { Multiplication factor, - } \\ p & \text { Probability, - } \\ \boldsymbol{P} & \text { Transition matrix, - } \\ \boldsymbol{\pi} & \text { Probability vector, - } \\ T & \text { Temperature, K } \\ \boldsymbol{U} & \text { Global transition matrix, - }\end{array}$

\section{Greek symbols}

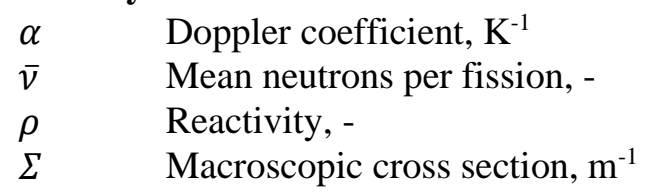

\section{Subscripts}

$\begin{array}{ll}c & \text { Capture } \\ C N & \text { Complex network } \\ c S & \text { Capture to scattering } \\ D & \text { Doppler } \\ f & \text { Fission } \\ f s & \text { Fission to scattering }\end{array}$




$\begin{array}{ll}M C & \text { Monte Carlo } \\ n r & \text { Non reaction } \\ s & \text { Scattering } \\ s c & \text { Scattering to capture } \\ s f & \text { Scattering to fission } \\ t & \text { Time step }\end{array}$

\section{References}

Aufiero, M., Brovchenko, M., Cammi, A., Clifford, I., Geoffroy, O., Heuer, D., Laureau, A., Losa, M., Luzzi, M., Luzzi, L., Merle, E., Ricotti, M.E., 2014. Calculating the effective delayed neutron fraction in the Molten Salt Fast Reactor: analytical, deterministic and Monte Carlo approaches, Annals of Nuclear Energy 65, 390401.

Barabási, A.L., 2016. Network Science, available at http://barabasi.com/book/network-science.

Calderoni, F., Brunetto, D., Piccardi, C., 2017. Communities in criminal networks: A case study, Social Networks 48, 116-125.

Cervi, E., Lorenzi, S., Cammi, A., Luzzi, L., 2017. An Euler-Euler multi-physics solver for the analysis of the helium bubbling system in the MSFR, NENE $201726^{\text {th }}$ International Conference for New Europe, Bled, Slovenia, September 11-14, 2017.

Cervi, E., Cammi, A., Di Ronco, A., 2018. Stability analysis of the Generation-IV nuclear reactors by means of the root locus criterion, Progress in Nuclear Energy 106, 316-334.

Cervi, E., Cammi, A., 2018. Stability analysis of the Supercritical Water Reactor by means of the root locus criterion, Nuclear Engineering and Design 338, 137-157.

Chiesa D., Clemenza, M., Nastasi, M., Pozzi, S., Previtali, E., Scionti, G., Sisti, M., Prata, M., Salvini, A., Cammi, A., 2016. Fuel burnup analysis of the TRIGA Mark II reactor at the University of Pavia, Annals of Nuclear Energy 96, 270-276.

DRAGON5 documentation available at http://www.polymtl.ca/phys/en/dragon-download.

Duran-Nebreda, S., Bassel, G.W., 2017. Bridging Scales in Plant Biology Using Network Science, Trends in Plant Science 22, 1001-1003.

Fionda, V., 2018. Networks in Biology, Reference Module in Life Sciences.

Fiorina, C., Hursin, M., Pautz, A., 2017. Extension of the GeN-Foam neutronic solver to SP3 analysis and application to the CROCUS experimental reactor, Annals of Nuclear Energy 101, 419-428.

Gan, C., Yang, X., Liu, W., Zhu, Q., Jin, J., He, L., 2014. Propagation of computer virus both across the Internet and external computers: A complex-network approach, Communications in Nonlinear Science and Numerical Simulation 19, 2785-2792.

Haghighat, A., 2014. Monte Carlo Methods for Particle Transport, CRC Press, Taylor \& Francis Group, Boca Raton, London, New York.

Hamieh, S.D., Saidinezhad, M., 2012. Analytical solution of the point kinetics equations with temperature feedback, Annals of Nuclear Energy 43, 148-152. 
Hayat, S., 2017. Computing distance-based topological descriptors of complex chemical networks: New theoretical techniques, Chemical Physics Letters 688, 51-58.

Hebért, A., 2016. DRAGON5 and DONJON5, the contribution of École Polytechnique de Montréal to the SALOME platform, Annals of Nuclear Energy 87, 12-20.

Holschuh, T.V., Marcum, W.R., 2018. Modified Fuchs-Nordeim model for use in reactor pulse measurements, Annals of Nuclear Energy 116, 314-318.

Lamarsh, J.R., 1969. Introduction to Nuclear Reactor Theory, Addison-Wesley Publishing Company.

Leitold, D., Vathy-Fogarassy, Á., Abonyi, A., 2017. Controllability and observability in complex networks the effect of connection types, available at https://www.nature.com/articles/s41598-017-00160-5.

Leppänen, J., Pusa, M., Viitanen, T., Valtavirta, V., Kaltiaisenaho, T., 2015. The Serpent Monte Carlo code: Status, development and application in 2013, Annals of Nuclear Energy 82, 142-150.

Liu, Y., Tan, Y., 2013. Complexity Modeling and Stability Analysis of Urban Subway Network: Wuhan City Case Study, Procedia - Social and Behavioral Sciences 96, 1611-1621.

Newman, M., 2010. Networks: An Introduction, Oxford University Press, London.

Orlova, Y., Kryven, I., Iedema, P.D., 2018. Automated reaction generation for polymer networks, Computers \& Chemical Engineering 112, 37-47.

Piccardi, C., 2011. Finding and Testing Network Communutues by Lumped Markov Chains, available at https://journals.plos.org/plosone/article?id=10.1371/journal.pone.0027028.

Schimit, P.H.T., Pereira, F.H., 2018. Disease spreading in complex networks: A numerical study with Principal Component Analysis, Expert Systems with Applications 97, 41-50.

Sun, Y., Liu, C., Zhang, C.X., Zhang, Z.K., 2014. Epidemic spreading on weighted complex networks, Physics Letters A 378, 635-640.

Tramm, J.R., Smith, K.S., Forget, B., Siegel, A.R., 2018. ARRC: A random ray neutron transport code for nuclear reactor simulation, Annals of Nuclear Energy 112, 693-714.

Raducha, T., Gubiec, T., 2017. Coevolving complex networks in the model of social interactions, Physica A: Statistical Mechanics and its Applications 471, 427-435.

Van Steen, M., 2010. Graph Theory and Complex Networks, published by Maarten van Steen.

Rowlands, J., Benslimane-Bouland, A., Cathalau, S., Giffard, F-X., Jacqmin, R., Rimpault, G., Bernnat, W., Mattes, M., Coste, M., Fernex, F., Van der Gucht, C., de Leege, P.F.A., Dean, C.J., Smith, N., Finck, P., Hogenbirk, A., Trkov, A., 1999. LWR pin cell benchmark intercomparison, JEFF Report 15, OECD-NEA.

Santamarina, A., Bernard, D., Blaise, P., Coste, M., Courcelle, A., Huynh, T.D., Jouanne, C., Leconte, P., Litaize, O., Ruggiéri, J-M., Sérot, O., Tommasi, J., Vaglio, C., Vidal, J-F., 2009. The JEFF-3.1.1 Nuclear Data Library, JEFF Report 22, OECD-NEA.

Woodcock, E.R., Murphy, T., Hemmings, P.J., Longworth, T.C., 1965. Techniques used in the GEM code for Monte Carlo neutronics calculations in reactors and other systems of complex geometry, ANL-7050, Argonne National Laboratory. 
614 Yang, L.X., Yang, X., Liu, J., Zhu, Q., Gan, C., 2013. Epidemics of computer viruses: A complex-network approach, Applied Mathematics and Computation 219, 8705-8717.

Yuan, X., Xue, Y., Liu, M., 2013. Analysis of an epidemic model with awareness programs by media on complex networks, Chaos, Solitons \& Fractals 48, 1-11.

Zañudo, J.G.T., Yang, G., Albert, R., 2017. Structure-based control of complex networks with nonlinear dynamics, Proceedings of the National Academy of Sciences of the United States of America 114, 7234-7239. network, Expert Systems with Applications 93, 200-211. 